\title{
Luca Bertolino*
}

\section{„Bach in die Synagogen!“. Erlösende Noten in Franz Rosenzweig}

https://doi.org/10.1515/naha-2019-0009

Published online September 24, 2020

Abstract: Starting with theoretical considerations on redemption in Rosenzweig's Der Stern der Erlösung, this paper highlights the connection between redemption and choral form in Church music (e.g. in Bach's Passions and in musical mass). Therefore, according to Rosenzweig, one can clearly distinguish between sacred/ religious (geistlich) and spiritual/intellectual (geistig) music. Rosenzweig also writes about renewing Jewish worship through Bach's vocal music, but we are given scant hints about this. "Bach in die Synagogen!" is nevertheless important, not only as an example for interreligious dialogue, but above all as an invitation to think about redemption in the postmodern condition.

Keywords: Franz Rosenzweig, redemption, music, postmodernity

Dieser Beitrag möchte die Aufmerksamkeit der Forschung auf die Musikrezensionen von Franz Rosenzweig lenken, die er in der fortgeschrittenen Phase seiner Krankheit verfasst hat, sowie auf ein angedachtes Projekt, zu dem es Aufzeichnungen gibt. Es handelt sich um kleinere, vielleicht sogar marginale Arbeiten, die jedoch eine bedeutende Bestätigung für theoretische Aspekte des mit Der Stern der Erlösung ${ }^{1}$ vertretenen „,neuen Denkens“2 ${ }^{\text {“2 }}$ ind. Vor allem aber liefern sie Anregungen, um über die Aktualität von Rosenzweigs theoretischem Vorschlag bzw. über die Aktualität der Philosophie überhaupt nachzudenken.

1 Franz Rosenzweig, Der Stern der Erlösung (Frankfurt a. M.: J. Kauffmann, 1921). Im Folgenden zitiert nach der 4. Aufl. (= Der Mensch und sein Werk. Gesammelte Schriften, II), hrsg. v. Reinhold Mayer (Haag: Martinus Nijhoff, 1976).

2 Vgl. Franz Rosenzweig, „Das neue Denken. Einige nachträgliche Bemerkungen zum ,Stern der Erlösung “, Der Morgen 1 (1925), Nr. 4, 426-451. Im Folgenden zitiert nach: ders., Zweistromland. Kleinere Schriften zu Glauben und Denken (= Der Mensch und sein Werk. Gesammelte Schriften, III), hrsg. v. Reinhold und Annemarie Mayer (Dordrecht/Boston/Lancaster: Martinus Nijhoff, 1984), 139-161.

*Corresponding author: Luca Bertolino, University of Turin, Department of Philosophy and Education Sciences, Turin, Italy, E-mail: luca.bertolino@unito.it. https://orcid.org/0000-00016749-7857 
Was das Thema „Erlösung“ angeht, bei dem die nachstehenden Reflexionen ansetzen, seien einleitend einige Angelpunkte meiner Interpretation dargelegt. Damit wird freilich kein Anspruch erhoben, die zahlreichen Fragen, die das Thema aufwirft, erschöpfend zu beantworten.

\section{Erlösung}

Die Erlösung ist in herausragender Weise Beziehung. Dies festzustellen bedeutet nicht, die zentrale Bedeutung der Offenbarung im Aufbau von Der Stern der Erlösung sowie als religiöse Erfahrung par excellence infrage zu stellen, denn sie ermöglicht den Zugang zur Beziehungsdimension, indem sie deren Grammatik und Zeitlichkeit lehrt und dadurch Orientierung bietet. ${ }^{3}$ Es bedeutet vielmehr, eine Folge dieser grundlegenden Offenheit zu betonen. Dass an der Erlösung nämlich nicht nur der Mensch und die Welt teilhaben, sondern auch Gott voll und ganz an ihr beteiligt ist, gestattet es, die Elemente der immerwährenden Vorwelt in ihrem Dreiecksverhältnis der allzeiterneuerten Welt zu erfassen. Dort sind sie in ihrem ontologischen In-sich-Sein und erkenntnistheoretischen Für-sich-Sein (in se esse et per se percipi $)^{4}$ abgesondert und daher nur erkennbar; hier dagegen sind sie der „Meta“-Dimension (Meta-physik, Meta-logik, Meta-ethik) ${ }^{5}$ entzogen und in ihrer wechselseitigen Beziehung erfahrbar. Sicher verweist Rosenzweig auch in der Schöpfung und in der Offenbarung insofern auf ein Dreiecksverhältnis der vorweltlichen Tatsächlichkeiten, als der Mensch, dem Gott seine Liebe offenbart, zum einen „das letztgeschaffene unter den [weltlichen] Geschöpfen“ und zum anderen, in der Schöpfung, Adam ist, d. h. „der erste Eigenname unter lauter Gattungsgeschöpfen, [...] wirklich ,im Ebenbilde Gottes' geschaffen“. ${ }^{6}$ Doch in der Erlösung sind Gott, die Welt und der Mensch in ihrer vollen Entfaltung gegeben, sodass darin „neben Ich-Du zwei ebenso wesenhafte Grundworte“ Ausdruck finden, „, in deren eine Hälfte sich jeweils ebensosehr das ganze Wesen der anderen Hälfte

3 Vgl. Franz Rosenzweig, ,„Urzelle“ des Stern der Erlösung. Brief an Rudolf Ehrenberg vom 18.XI. 1917“, in: ders., Kleinere Schriften (Berlin: Schocken Verlag/Jüdischer Buchverlag, 1937), 357-372. Im Folgenden zitiert nach: ders., Zweistromland, 125-138: $125 \mathrm{f}$.

4 Vgl. Rosenzweig, „Das neue Denken“, $144 \mathrm{f}$.

5 Vgl. Rosenzweig, Der Stern der Erlösung, Erster Teil.

6 Ebd., 172. Vgl. 1. Mose 1,26. 
hineingießt wie bei Ich-Du [...]: ER-Es [...], Wir-ES“7 - um hier an die Lösung zu erinnern, die Rosenzweig Martin Buber vorschlug, um die Reduktion der Schöpfung auf ein totes Es, d. h. auf einen Denkgegenstand, zu vermeiden. Denn der von Gott in der Offenbarung geliebte Mensch (ICH-Du), dem damit das Gebot „Liebe mich“ auferlegt ist (Ich-DU), richtet seine Liebestat auf das Reich der Welt (Wir-ES) als ewig wachsende Kreatur Gottes (ER-Es), genau genommen auf seinen Nächsten (vgl. 3. Mose 19,18) als Platzhalter der Welt. ${ }^{8}$ So erlösen und binden Mensch und Welt einander gleichzeitig, aber nur Gott ist der Erlöser im engeren Sinn: der Erlöser der Welt durch den Menschen, dem er sich offenbart, der Erlöser des Menschen in der von ihm geschaffenen Welt und der Erlöser seiner selbst in der Erlösung beider. ${ }^{9}$

Die Erlösung ist ein zukünftiges zeitliches Ereignis. Es steht neben anderen zeitlich konnotierten Ereignissen: der Vergangenheit der Schöpfung und der Gegenwart der Offenbarung. Sie sind jedoch nicht in einer unterschiedenen Form gegeben, sondern immer in einer „Bahn“10 oder „Reihe“11, die ein Ganzes darstellt: im Plexus Schöpfung-Offenbarung-Erlösung. Denn der gegenwärtige Augenblick, in dem Gott den Dialog mit dem Menschen allzeit erneuert, bringt die vergangene Weltschöpfung, die durch das Wort Gottes geprägt ist, mit sich und erschließt die Erlösung als ewige Zukunft des Gottesreichs, das Mensch und Welt im gemeinsamen Gebet vorwegnehmen können. Umgekehrt - so behauptet Rosenzweig - ist „die Offenbarung selbst und ihre Einbindung und Begründung in die Zuversicht auf das Kommen des sittlichen Reichs der endlichen Erlösung [...] einzubauen in den Begriff der Schöpfung““, ${ }^{12}$ so wie „die Erlösung [...] zur Schöpfung nicht intimer als zur

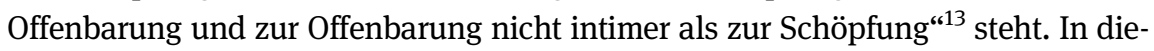
sem „trame ou [...] drame de l'être“ (Gewebe oder Drama des Seins) ${ }^{14}$ - dies der bekannte, treffende Ausdruck von Emmanuel Levinas - bezeichnen Schöpfung-

7 Franz Rosenzweig, Briefe und Tagebücher (= Der Mensch und sein Werk. Gesammelte Schriften, I), 2 Bde., hrsg. v. Rachel Rosenzweig und Edith Rosenzweig-Scheinmann unter Mitwirkung v. Bernhard Casper (Haag: Martinus Nijhoff, 1979), Bd. 2, 824-827 (undatierter Brief an Martin Buber, den Rosenzweig im September 1922 schrieb): 825 f. Dazu vgl. Bernhard Casper, „Franz Rosenzweigs Kritik an Bubers ,Ich und Du““, Philosophisches Jahrbuch 86 (1979), Hbd. 2, 225-238; jetzt in: ders., Religion der Erfahrung. Einführungen in das Denken Franz Rosenzweigs (Paderborn/München/Wien/Zürich: Ferdinand Schöningh, 2004), 101-116.

8 Vgl. Rosenzweig, Der Stern der Erlösung, 238 f., 243.

9 Vgl. ebd., $254 \mathrm{f}$.

10 Ebd., 101.

11 Ebd., $210 \mathrm{f}$.

12 Ebd., 114.

13 Ebd., 257.

14 Emmanuel Levinas, „Franz Rosenzweig: une pensée juive moderne“, Revue de Théologie et de Philosophie 98 (1965), Nr. 4, 208-221: 213. 
Offenbarung-Erlösung also nicht einzelne Begriffskategorien oder die geschichtlich sich entfaltende, streng zeitliche Abfolge von Vergangenheit-Gegenwart-Zukunft. Vielmehr stehen sie für die Fülle der Wirklichkeit, in der alles geschieht. Die Reihe wirft offensichtlich Interpretationsschwierigkeiten auf, die sich aus der Unfähigkeit des philosophischen Blickes ergeben, die vielfältigen Fäden, die das Geflecht bilden, synoptisch zu begreifen - um die Metapher Levinas' hier wieder aufzunehmen. Ganz zu schweigen von der Unfähigkeit des philosophischen Schreibens, bei dem - wie Rosenzweig selbst uns lehrt - tausend Verbindungen des Denkens „fein säuberlich auf die Schnur von Tausenden Zeilen gereiht werden" ${ }^{15}$ müssen. Die größte Schwierigkeit besteht allerdings darin, die Schöpfung-Offenbarung-Erlösung in ihrer Ereignisdimension zu erfassen, d. h. nicht als objektiv erkennbares Leben, sondern als subjektives Erlebnis.

Die Erlösung enthüllt ein überhistorisches Szenario. Wenn die Wirklichkeit in der allzeiterneuerten Welt voll erfahrbar ist, kommt sie jedoch nicht ohne einen zweifachen Bezug aus: zum einen den Bezug auf die immerwährende Vorwelt der Elemente, die reine Tatsächlichkeiten, aber zugleich notwendige Voraus setzungen sind, und zum anderen den Bezug auf die ewige Überwelt, in der Mensch und Welt ihre Erlösungsaufgabe erfüllen, um zur Schau der Wahrheit als leuchtendes Antlitz Gottes zu gelangen. Anders gesagt: Die drei Teile von Der Stern der Erlösung mit ihren unterschiedlichen Szenarien sind durch eine Metaphysik der Zeit geprägt und miteinander verbunden, nämlich durch die mythische Zeitlo-sigkeit, die Ereigniszeit des Plexus Schöpfung-Offenbarung-Erlösung und die unbegrenzte Zeit des Reichs. Im Rahmen der innerweltlichen Zeitereignisse und der überhistorischen ewigen Szenarien nimmt das Erlösungshandeln von Mensch und Welt die Ewigkeit vorweg.

Dies ist im Übrigen ein Aspekt, der nach Rosenzweig das Judentum wie das Christentum kennzeichnet und einander annähert, wie er im dritten Teil von Der Stern der Erlösung erklärt: Mit der zyklischen Wiederkehr ihrer liturgischen Feste halten sie die Ewigkeit im linearen Zeitverlauf fest; im jeweiligen Gemeinschaftsgebet „zur rechten Zeit und am rechten Ort“16 erbeten sie das Reich Gottes; das Judentum, aus aller Weltgeschichte herausgestellt, garantiert die Ewigkeit der Verheißung, während die um das Ereignis Christi gesammelte ekklēsia in der historischen Zeit von der Verwirklichung der Ewigkeit Zeugnis ablegt. Den hermeneutischen Schlüssel, um diesen Teil des Werkes sowie die früheren Teile zu verstehen, liefert der Titel der Einleitung. Untersucht wird letztlich immer dieselbe Ganzheit; was sich ändert, ist die Methode, mit der man sich ihr mit je besonderen Möglichkeiten und unterschiedlichen Grenzen nähert (erkennen, erfahren, erbeten), sowie folglich die Form, in der diese Ganzheit sich präsentiert (als All,

15 Rosenzweig, „Das neue Denken“, 142.

16 Rosenzweig, Der Stern der Erlösung, 465. 
Wunder, Reich). Das Erbeten lädt zu einer zugleich individuellen und kollektiven Handlung ein. In diesem Zusammenhang ist daran $\mathrm{zu}$ erinnern, gegen wen Rosenzweig sich wendet: Ausgehend von den doctores über die fideles und die clerici ermittelt er nämlich die tyranni als Zielscheibe der Polemik. ${ }^{17}$ Kurioserweise ist „in tyrannos!“18 auch das Motto, das Rosenzweig auf den Seiten eines Stammbuchs fand, das während der Studienzeit am Tübinger Stift Hegel gehört hatte („Vive la liberté! Vive Jean Jacques! In Tyrannos!“ - handschriftlich von seinen Freunden mit offenkundigem Bezug auf den aufklärerischen Geist der Epoche verfasst). ${ }^{19}$ In Der Stern der Erlösung stehen die „Tyrannen“ jedoch nur für die „Schwärmer“ und „Sektierer“, ${ }^{20}$ d. h. diejenigen, die dem Nächsten, der die Welt repräsentiert, das Übernächste als Himmelreich vorziehen und Letzterem dergestalt Gewalt antun und sein Wachstum verhindern.

Auf welche Weise kann der Mensch eine Beziehung zur Welt unterhalten, die das Reich Gottes vorwegnimmt und im konkreten Erleben in der Gegenwart die kommende Zukunft erschließt? Die sich der Anschauung der ewigen Wahrheit öffnet und den Nächsten achtet, ohne ihn zu überwinden? Bekanntlich erblickt Rosenzweig in der Liturgik, vor allem in der gemeinsamen Gebärde, das Organon des Erbetens. An anderer Stelle wurden von mir sowohl die Bedeutung des Chores der Erlösung, in dem Mensch und Welt als Wir sich in einem Lobgesang für Gott vereinen, als auch die Bedeutung des Chores in der Kirchenmusik bereits herausgestellt. $^{21}$ Auf den musikalischen Aspekt wird im Folgenden näher eingegangen. ${ }^{22}$

17 Vgl. Franz Rosenzweig, Die „Gritli“-Briefe. Briefe an Margrit Rosenstock-Huessy, hrsg. v. Inken Rühle und Reinhold Mayer (Tübingen: BILAM Verlag, 2002), 137 f., 196 f., 199 f., 277 f. (Briefe an Margrit Rosenstock vom 31. August, 20. November, 26. November 1918 bzw. 14. April 1919): 138, 196, 200 bzw. 278; ders., Briefe und Tagebücher, Bd. 2, 603-605 (Brief an Rudolf Ehrenberg vom 4. September 1918): 604 sowie 607 f. (Brief an Gertrud Oppenheim vom 25. September 1918): 608.

18 Rosenzweig, Der Stern der Erlösung, 295.

19 Vgl. Franz Rosenzweig, Hegel und der Staat, 2 Bde. (München/Berlin: R. Oldenbourg, 1920), Bd. 1, 18. 20 Rosenzweig, Der Stern der Erlösung, 302.

21 Siehe Luca Bertolino, „Die Rolle des Chores in Franz Rosenzweigs Stern der Erlösung“, Rosenzweig Jahrbuch / Rosenzweig Yearbook 5 (2010), 141-159.

22 Insbesondere haben sich dem Thema „Musik“ bei Rosenzweig zugewandt: Francesca Albertini, „Musik, Poesie, Offenbarung. Die Ästhetik Franz Rosenzweigs“, Musik \& Ästhetik 4, H. 15 (2000), 5567; Arnold Jacob Wolf, „Franz Rosenzweig and Religious Music“, Judaism. A Quarterly Jourmal of Jewish Life and Thought 49 (2000), Nr. 4, 479-483; Thomas Eicker, Einsäen der Ewigkeit ins Lebendige. Impulse der Ästhetik Franz Rosenzweigs für eine Theologie gottesdienstlicher Musik (Paderborn/München/Wien/Zürich: Ferdinand Schöningh, 2004), bes. 235-322; Klaus-Jürgen Sachs, „Musik - erfahren und erörtert durch Franz Rosenzweig (1886-1929)“, in: Musik - Zu Begriff und Konzepten. Berliner Symposion zum Andenken an Hans Heinrich Eggebrecht, hrsg. v. Michael Beiche und Albrecht Riethmüller (Stuttgart: Franz Steiner, 2006), 87-113; ders., „Musik im Denken Franz Rosenzweigs“, in: Franz Rosenzweigs „neues Denken“. Internationaler Kongreß Kassel 2004, 2 Bde., 


\section{Musik}

Zunächst sei hervorgehoben, dass die Kunst - sei sie tönend, bildend oder darstellend - nach Rosenzweig nur dann eine authentische, organische Beziehung zum Leben herzustellen vermag, wenn sie angewandt wird. Anderenfalls, in ihrer Reinheit, setzt sie den Menschen der „Krankheit der Weltentfremdung، ${ }^{23}$ aus, weil sie ihn dazu verurteilt, in einem hortus conclusus zu leben, der ein Surrogat des göttlichen gan 'Eden ist. So ersetzt der Idealismus, der den Sündenfall begangen hat, die Logik der Sprache vorzuziehen - dies Rosenzweigs Vorwurf -, die Lebendigkeit der Schöpfung durch die Vergötterung des Kunstwerks. Er begreift das Kunstwerk mit anderen Worten als unbewusstes Produkt des Geistes und erhebt es dergestalt zur sichtbaren Erscheinung eines Absoluten. ${ }^{24}$ Statt idealistisch dem reinen Kunstgenuss nachzustreben und in einem menschlichen Garten zu leben, muss das Kunstwerk also der Konkretheit eines Raumes und einer Zeit und lebhaften Gebärden zurückgegeben werden, in denen und mit denen der Mensch sich Gott erneut annähern kann. In der liturgischen Anwendung wird die Kunst genau von der idealistischen Reinheit gereinigt und erlaubt es dem Menschen, ins Paradies der ewigen Überwelt einzutreten.

Die Kirchenmusik stellt sich insofern als Antizipation der Erlösung dar, als sie dem Menschen das Geleit gibt, sodass er sich „mit beiden Beinen in die Zeit, in die allerwirklichste Zeit, in die eine Zeit des einen Welttags, dessen alle einzelnen Welttage nur Teile sind, hineinstell[t]““. ${ }^{25}$ Denn durch seine Anwendung der Musik auf die Feste des liturgischen Kalenders bewirkt das Christentum, dass die musikalische Melodie vom Zuhörer nicht, sich in der Illusion eines idealen Lebens wiegend, vereinzelt genossen wird, sondern gemeinschaftlich in der wirklichen Zeit. In Der Stern der Erlösung unterscheidet Rosenzweig nämlich klar zwischen frevelhafter und heiliger Musik. Während Erstere in den Konzertsälen gehört wird und von einer individuell idealen Zeit gekennzeichnet ist, wird Letztere in den Kirchen erlebt und zeichnet sich durch die von der Offenbarung mit ihrem Rückverweis auf die Schöpfung und ihrem Vorverweis auf die Erlösung in der

hrsg. v. Wolfdietrich Schmied-Kowarzik (Freiburg/München: Karl Alber, 2006), Bd. 1, 446-455; Evan Kent, „California Dreamin': Franz Rosenzweig Goes to Jewish Summer Camp“, Musiikkikasvatus. The Finnish Journal of Music Education 19 (2016), Nr. 2, 8-16; Gianfranco Bonola, „Rosenzweig infermo e la musica tra fruizione, mercato e significato“, L'ospite ingrato NS 4 (2017), 103-106; Luca Bertolino, „Religion und Musik bei Franz Rosenzweig“, in: Religion.Geist.Musik. Theologisch-kulturwissenschaftliche Grenzübergänge, hrsg. v. Hans Martin Dober und Frank Thomas Brinkmann (Wiesbaden: Springer VS, 2019), 59-84.

23 Rosenzweig, Der Stern der Erlösung, 393.

24 Vgl. ebd., $162 \mathrm{f}$.

25 Ebd., 401. 
Wirklichkeit gesetzte zeitliche Orientierung aus. Es geht also darum, die Musik den Konzertsälen zu entziehen, sie vom subjektiven Genuss zu reinigen, der leicht zu einer aufgeregten Selbstzufriedenheit sowie zur Selbstvergessenheit führen kann, und sie dank ihrer Einbindung in die Liturgie in die reale Zeit der Welt einzubetten, d. h. ins Leben zurückzuführen.

Als Modell und sogar als „eigentliche Grundlage der kirchlichen Anwendung der Musik“ ${ }^{26}$ schwebt Rosenzweig der Choral vor, vor allem die Choräle der BachPassionen, aber auch der gregorianische Choral der katholischen Kirchenmusik. In der Einmütigkeit des Chors wird zuerst das besondere Wort jedes Einzelnen in seiner Eigenheit zum Schweigen gebracht. In der Harmonisierung des Chorals werden dann die einzelnen Stimmen in der Einheit einer nicht kontrapunktischen Polyfonie vereint; obgleich die verschiedenen Melodielinien unabhängig bleiben, erklingen sie im Einklang und werden Ausdruck der Zusammengehörigkeit aller Versammelten. Außerdem stimmt der Choral nicht nur die einzelnen Chorstimmen aufeinander $\mathrm{ab}$, indem er sie auf die wirkliche Gemeinschaft des Wir vorstimmt, sondern erleichtert auch die gemeinsame Sammlung derjenigen, die diese Musik nur mithören und nicht mitsingen. Es handelt sich insbesondere um die Teilnehmer an der musikalischen Messe, die - ein jeder für sich - zum Gleichklang der Gefühle und der gemeinsamen Worte zur Musik bewogen werden. Schließlich werden selbst die Worte, obwohl sie für sich auch wirkungsvoll sind, durch ihr Eingehen in den Choral der Kirchenliturgie nach Rosenzweig zeitweilig verwandelt:

Das Wort, das zum Text des Gesangs geworden ist, hört auf, ein beliebiges zu sein. Mit Sangweisen erhalten sich Worte [...]. Lebendig sind sie schon. Aber ihre Lebendigkeit ist vergänglich. Indem die Musik sie aufnimmt, werden sie dauerhaft. Und wenn die Musik Kirchenmusik ist, dann [...] werden [sie] durch die Eingliederung in den ewigen Tag des Herrn selber ewig. ${ }^{27}$

Auch in den Schallplattenrezensionen, die Rosenzweig zwischen Frühjahr 1928 und Herbst 1929 im Feuilleton des Kasseler Tageblatts veröffentlichte, bestätigt er die Bedeutung der musikalischen Messe und der anderen neben ihr erblühten Formen liturgischer Musik. Insbesondere die Einwebung von Solostimmen in den Chor, die er beispielsweise an dem Schlusschor und Terzett von Teil 1 des haydnschen Oratoriums Die Schöpfung (Hob. XXI:2, Nr. 14: „Die Himmel erzählen die Ehre Gottes“; vgl. Ps 19,1-3) schätzt, ist nach Rosenzweig nicht nur ein technischer Fortschritt, sondern deutet auch auf die vollständige Verwirklichung des Wir hin, das durch sein Gebet die Erlösung vorwegnimmt:

26 Ebd., 402.

27 Ebd., 403. 
Das Wir der betenden Gemeinde nährt sich von dem hundertfachen Ich ihrer Glieder, doch die können nur „Ich“ sagen, weil das „Wir“ der Gemeinde sie trägt. Diese intimste wechselseitige Verflechtung von mir und allen findet jetzt ihre Ausdrucksform. Und damit ist das höchste Werk der ganzen Gattung, die noch in Bachs H-Moll-Messe nicht über die großartige Objektivität eines bildhaften Nebeneinanders der Chöre und der Solostücke hinauskam, möglich geworden: Beethovens Missa solemnis. ${ }^{28}$

\section{Erlösende Noten}

Die Betrachtungen, die Rosenzweig zu unterschiedlichen Zeiten über die Missa solemnis D-Dur (op. 123) anstellte, lassen die Verbindung von Erlösung und Musik deutlicher hervortreten. In Der Stern der Erlösung unterstreicht der Autor polemisch die Vehemenz des „Credo“, das „die Worte von der vita venturi saeculi in immer neuen Wiederholungen herausjubelt, als ob sie Krone, Sinn und Bestätigung des ganzen Glaubens wären“. ${ }^{29}$ Es ist in gewissem Sinn ein „,aufklärerischer“ Glaube, der Idee vom „Fortschritt“ der Menschheit verpflichtet, die mit Schleiermacher das lutherisch gegenwärtige Erleben des gläubigen Gefühls an die Hoffnung auf das zukünftige Reich der Sittlichkeit knüpft. Man könnte sagen, dass „,die nicht endende Ausspinnung des ,Lebens der kommenden Welt' gegen die kurz erledigte Auferweckung der Toten " ${ }^{\text {“30 }}$ in der musikalischen Komposition den Sieg des menschlichen Paradieses über das göttliche anzeigt. In „Der Konzertsaal auf der Schallplatte“ nimmt Rosenzweig dagegen von seiner früheren Interpretation Abstand und bestätigt mit Nachdruck den geistlichen Charakter der Missa solemnis, wobei er dazu auffordert, sie den Konzertsälen zu entziehen, wo sie gewöhnlich auch aufgrund ihres Umfangs aufgeführt wurde. Dabei geht er so weit, die Kirchenmusik mit der attischen Tragödie zu vergleichen:

An sich würde es [das Werk Missa solemnis] grade durch die Einflechtung der Solostimmen die stärkste Wirkung dort [in der Kirche] ausüben können, - eine Wirkung, die mit seiner uns aus den Konzertsälen gewohnten so unvergleichbar wäre wie die Wirkung der Antigone auf den athenischen Hörer mit der des Buchs oder auch einer Aufführung auf uns. ${ }^{31}$

28 Franz Rosenzweig, „Der Konzertsaal auf der Schallplatte“, Kasseler Tageblatt 75 (1928), A Nr. 223 / B Nr. 132, 13. Mai; A Nr. 255 / B Nr. 152, 3. Juni; Nr. 197, 18. Juli; Nr. 245, 4. September; Nr. 301, 30. Oktober; sowie 76 (1929), A Nr. 45 / B Nr. 27, 27. Januar; A Nr. 160 / B Nr. 95, 7. April; Nr. 197, 19. Juli; Nr. 324, 23. November. Im Folgenden zitiert nach: ders., Zweistromland, 427-448: 435.

29 Rosenzweig, Der Stern der Erlösung, 111.

30 Rosenzweig, „Der Konzertsaal auf der Schallplatte“, 435.

31 Ebd., $435 \mathrm{f}$. 
Darüber hinaus klärt Rosenzweig anhand einer Gegenüberstellung der Missa solemnis und der beethovenschen Musikbearbeitung von Schillers Gedicht „An die Freude“ im Schlusssatz der 9. Sinfonie d-Moll (op. 125) Ähnlichkeiten und Unterschiede zwischen geistlicher und weltlicher Musik bzw. zwischen geistlichem und geistigem Gesang. Im Wettstreit mit der geistlichen Musik will die gegen 1800 entstandene „Musik des Geistes“ „,sich [...] ebenso weltüberfliegend[...] und dann freilich nicht weltüberwindend[...], sondern weltverklärend[...]“ geben, indem sie „den Konzertsaal als Raum für ihr Erklingen verlangt, eben weil er ein neutraler Raum ist, der jedem offen steht“ “ ${ }^{32}$ In diesem Sinn ruft die Schallplattenaufnahme der englischen Aufführung der Ode durch den London Philharmonic Choir unter der Orchesterleitung von Albert Coates ${ }^{33}$ in Rosenzweig „ein[en] fast erschütternde[n] Eindruck“34 hervor: „ein ganz unmittelbares Erlebnis der einen Menschheit“. Etwas „ähnliches müssen die ersten empfunden haben, denen die Bibel im Gewand einer neuen Sprache entgegentrat““35 - fügt Rosenzweig hinzu und erläutert auf diese Weise auch die Bedeutung seines 1925 zusammen mit Martin Buber begonnenen Projektes, den deutschsprachigen Juden eine Verdeutschung der Schrift vorzulegen. Man kann also in der weltlichen Musik einem ähnlichen Miteinandersingen des Ich und des Wir begegnen wie in der Kirchenmusik. Bei genauerer Betrachtung ist ihre Grundform jedoch radikal verschieden. Im geistlichen Gesang löst sich die Stimme aus dem Chor - „etwa gleich in den ersten Takten der Missa“, kommentiert Rosenzweig -, während sie im geistigen Gesang der Ode den Chor aufruft und erweckt: „Dort Menschheit vor Gott, hier Mensch in Menschheit - und Gott nur geahnt, nur gesucht, nur ,überm Sternenzelt"“ 36

Neben der vorstehend skizzierten Unterscheidung zwischen weltlicher und geistlicher Musik verdient als weiteres Beispiel erlösender Noten Erwähnung, dass Rosenzweig auch eine musikalische Erneuerung des jüdischen Gottesdienstes vorschwebte. Durch einen Brief an Hermann Geiger vom 31. Dezember 1926 wissen wir von „einer alten Lieblingsidee von [ihm], der Gewinnung Bachscher Vokalmusik

32 Ebd., 436.

33 Es lässt sich nicht leicht feststellen, auf welche Aufnahme Rosenzweig Bezug nimmt: Ludwig van Beethoven, Symphony No. 9 in D minor, Op. 125, Symphony Orchestra, Leitung: Albert Coates, The Gramophone Co. HMV D 842-849, 1923, 78 min $^{-1}$, oder The Gramophone Co. HMV D 1164-1171, 1926, $78 \mathrm{~min}^{-1}$; jetzt unter: https://sounds.bl.uk/Classical-music/Beethoven/026M-1CL0029671XX-0100V0 bzw. https://sounds.bl.uk/Classical-music/Beethoven/026M-1CL0030211XX-0100V0. Berücksichtigt man, was er über den „,ganz helle[n], beinah tenorale[n] Bariton“ schreibt (Rosenzweig, „Der Konzertsaal auf der Schallplatte“, 436) - was jedoch schon in der Partitur zu lesen ist -, könnte es sich um diejenige von 1926 handeln.

34 Ebd.

35 Ebd., 437.

36 Ebd. Der Ausdruck „überm Sternenzelt“ ist ein Zitat aus der schillerschen Hymne. 
für den jüdischen Gottesdienst““. ${ }^{37}$ Diese Idee übertrug sich am 18. August 1928 in eine Anregung vor der jüdischen Jugend, als Rosenzweig den Brief mit dem programmatischen Titel „Bach in die Synagogen! Zur Reform der Reform“38 in der Zeitschrift Die Gemeinschaft. Hefte für die religiöse Erstarkung des Judentums publizierte. Die Hinweise, die er dazu gibt, sind spärlich. Es gehe darum,

im Gottesdienst neben dem ständigen Synagogenchor eine Bachsche Motette oder Kantate in ihren für die Synagoge geeigneten Partien im Gottesdienst aufzuführen [...]. Ganz kann ja das meiste nicht genommen werden, weil irgendeine Arie mindestens gewöhnlich die christliche Moral von der alttestamentlichen Geschicht zieht. Aber es gibt eine Fülle alttestamentlicher Texte, und grade die schönsten, in Bachscher Vertonung. Natürlich müßte, damit die Sache kein „Synagogenkonzert“ wird, sondern wirklich im Rahmen des Gottesdienstes bleibt, der Text einer sein, der sowieso an dem betreffenden Tag vorgelesen wird entweder aus der jeweiligen Perikope des Pentateuchs oder aus dem zugehörigen Profetenabschnitt. ${ }^{39}$

Die in der Jüdisch-liberalen Zeitung erschienene Nachricht über einen Vortrag zum Thema „Synagoge oder Konzertsaal“, den Geiger im Rahmen eines Heimabends beim Vorsitzenden der Frankfurter Jüdisch-liberalen Jugendgemeinschaft hielt und der dem jungen Musikstudenten ermutigende Worte Rosenzweigs eintrug, gestattet es, den kulturellen Kontext, in dem Rosenzweigs Gedanken Gestalt annahmen, sowie die Richtung dieser Gedanken genauer zu verstehen. Geiger

wies auf das Theatermäßige, Aeußerliche mancher Gottesdienste hin, beanstandete den Mangel an Andacht und das Verhalten der Gemeindemitglieder in den Gotteshäusern und hielt eine Renovierung des Gottesdienstes in modernem Sinne für erforderlich.

Einen Weg hierzu sah er in der Darbietung anderer Musik als der bisher üblichen und in einer künstlerischen Form. Er schlug vor, Bach und Händel, die Allgemeingut jedes religiösen Menschen seien, sprechen zu lassen; die mechanischen deutlichen Gebete auszuschalten und dafür dem Chor und dem freien Orgelspiel einen größeren Raum zu gewähren. $\mathrm{Zu}$ der Diskussion wurde als Gegengrund darauf hingewiesen, daß gerade in der Wiederholung derselben Gebete etwas Liebgewohntes und Vertrautes liege, wodurch bei den meisten Menschen das religiöse Gefühl vertieft würde; es wurden allerdings viele Mißstände erwähnt.

Schließlich einigte man sich dahin, daß der alte Gottesdienst mit wenigen Aenderungen beizubehalten sei, daneben etwas aber für die, die bisher fremd und

37 Rosenzweig, Briefe und Tagebücher, Bd. 2, 1119 f.: 1119.

38 Franz Rosenzweig, „Bach in die Synagogen! Zur Reform der Reform“, Die Gemeinschaft. Hefte für die religiöse Erstarkung des Judentums, 18. August 1928, Nr. 13/14, 20. Im Folgenden zitiert nach: ders., Briefe und Tagebücher, Bd. 2, $1119 \mathrm{f}$.

39 Ebd. 
lau dem Gottesdienst gegenüberstanden, geschaffen werden müsse. Dieses Neue könne am besten dadurch entstehen, daß die Jugend aus sich heraus Gottesdienste, Andachts- und Feierstunden abhalte, bei denen sie in künstlerischer Form oder auch in freier Rede Gelegenheit habe, ihrem Empfinden nach Gottesdienst zu halten. Diese Einrichtung auch in Frankfurt, wie sie schon in anderen Städten besteht, zu schaffen, sei die Aufgabe der liberalen Jugend.

Die Diskussion bewies durch ihre Lebhaftigkeit, wie brennend diese Fragen für unsere Jugend sind, und wie allenthalben das Bedürfnis besteht, das Alte, Wertvolle in eine [Edition: einer] dem modernen Empfinden leichter eingehende [Edition: eingehenden] und besser liegende Form zu kleiden, um wahrhaft religiös zu sein und zu werden. ${ }^{40}$

Im Reformjudentum, das nebenbei gesagt von Geigers Großvater, dem Rabbiner Abraham, angestoßen wurde, wurde die Form der Synagogenmusik also infrage gestellt. Dies ging so weit, dass sich der Oberkantor der Stuttgarter Synagoge, der Rabbiner Leo Adler, im Januar 1926 fragte, ob die Tatsache, dass in kürzester Zeit vier Artikel in drei jüdischen Zeitungen zum Thema der „Synagogalen Musik“ erschienen, rein zufällig sei, ob sie in Bezug auf eine bevorstehende Kantorenversammlung verstanden werden solle oder etwa als Einladung, diesen Fragenkomplex zu untersuchen. ${ }^{41}$ Augenscheinlich eröffnet sich damit ein vielversprechender Forschungsweg, der noch $\mathrm{zu}$ beschreiten ist und der $\mathrm{zu}$ den Kompositionen von Salomon Sulzer und Louis Lewandowski zurückführt. Zusammen mit diesem einführenden Hinweis sind mindestens auch die Beiträge von Dieter Adelmann zur synagogalen Chormusik des 19. Jahrhunderts zu erwähnen, ${ }^{42}$ vor allem insofern

40 Jüdisch-liberale Jugendgemeinschaft 6 (1926), Nr. 21 (21. Mai), 3.

41 Vgl. Leo Adler, „Synagogale Musik“, Jüdisch-liberale Zeitung 6 (1926), Nr. 2 (8. Januar), 7.

42 Vgl. Dieter Adelmann, „Schir Zion“ und „Hat die synagogale Chormusik des 19. Jahrhunderts eine religionsphilosophische Bedeutung?“, in: ders., „Reinige dein Denken“. Über den jüdischen Hintergrund der Philosophie von Hermann Cohen, hrsg. v. Görge K. Hasselhoff (Würzburg: Königshausen \& Neumann, 2010), 34-52 bzw. 94-104. Die Reflexionen von Rosenzweig über die bachsche Musik entstanden außerdem in einem bestimmten geistig-historischen Milieu, das sich mit der deutschen Kultur insgesamt auseinandersetzte. Die Bach-Rezeption seitens der deutschjüdischen Intellektuellen würde in diesem Sinn eine vertiefende Untersuchung verdienen, die freilich nicht Gegenstand dieses Beitrags sein kann. Als Beispiel sei etwa an Hermann Cohens Feststellungen in Ein Bekenntnis in der Judenfrage (Berlin: Ferd. Dümmler, 1880) erinnert; jetzt in: ders., Jüdische Schriften, 3 Bde., mit einer Einleitung v. Franz Rosenzweig, hrsg. v. Bruno Strauß (Berlin: C. A. Schwetschke \& Sohn, 1924), Bd. 2, 73-94: 80: Die Wiederentdeckung der MatthäusPassion (BWV 244) durch Felix Mendelssohn mit seiner Aufführung am 11. März 1829 in der Berliner Singakademie (102 Jahre nach der Uraufführung vom 11. April 1727 in der Leipziger Thomaskirche) sei einer Bewegung des jüdischen Gemütes zur geistlichen Musik Bachs zuzuschreiben. Diese Bewegung entspringe nicht nur der ästhetischen Rührung, sondern auch einer Gemeinsamkeit der religiösen Gefühle. Dagegen meinte Cohen 32 Jahre später in seinem System der Philosophie. Dritter Teil. Ästhetik des reinen Gefühls, 2 Bde. (Berlin: Bruno Cassirer, 1912), dass 
dieser nicht zuletzt der kulturgeschichtlichen und religionsphilosophischen Bedeutung sein Augenmerk schenkt. Die gleiche Aufmerksamkeit hatte im Übrigen Rosenzweig, dessen Vorschlag, die bachsche Musik in den jüdischen Gottesdienst einzubinden, nicht nur zum jüdisch-deutschen Dialog hätte beitragen können, sondern wiederum die zentrale Bedeutung der erlösenden Erfahrung unterstreicht, die von und in der Musik gefasst werden kann und muss. Ohne zu vergessen, dass nur die religiös angewandte Musik die Partitur lebendig machen kann. Kurz: Bach in den Synagogen

wäre ein rechtes Glück [...] für beide Beteiligten: für die Synagoge, die damit endlich statt braver Kapellmeistermusik Teil gewänne an der wirklichen großen deutschen Musik, aber auch ein Glück für Bach, denn diese Sachen schlafen ja größtenteils den Schlaf der Partitur, aus dem sie dann zu klingendem Leben erweckt würden. ${ }^{43}$

\section{Erlösende Noten heute}

Man könnte Rosenzweigs Vorschlag als eine der zahlreichen Vermischungen, die die Geschichte der Musik - nicht nur der heiligen - gekennzeichnet haben, ad acta legen. Oder man könnte den problematischen Charakter, wenn nicht gar die Unzeitgemäßheit seines Vorschlags in der heutigen Zeit unterstreichen, ${ }^{44}$ wenn man bedenkt, dass der gregorianische Gesang, der polyfone Gesang und sogar der Orgelklang, wenigstens in der katholischen Liturgie, zunehmend aufgegeben

„es [...] schlechthin ästhetische Barbarei [ist], wenn man diesen musikalischen Wert [die Matthäus-Passion] auf den Glaubenswert des Textes gründet“; jetzt in: ders., Werke, hrsg. v. Hermann-Cohen-Archiv am Philosophischen Seminar der Universität Zürich unter der Leitung v. Helmut Holzhey, Bd. 8-9, Einleitung v. Gerd Wolandt (Hildesheim/Zürich/New York: Georg Olms, 2005), Bd. 9, 161. Vgl. ferner die Erinnerung von Martin Buber, in: ders., Begegnung. Autobiographische Fragmente (Stuttgart: Kohlhammer, 1960), an den Einfluss der bachschen Musik, als er sie mit zwanzig Jahren in Leipzig hörte, der so groß war, dass „offenbar [...] der Grundton [s]eines Lebens irgendwie modifiziert [wurde] und erst von da aus auch der Gedanke“; jetzt in: ders., Werkausgabe, im Auftrag der Philosophischen Fakultät der Heinrich Heine Universität Düsseldorf und der Israel Academy of Sciences and Humanities hrsg. v. Paul MendesFlohr und Bernd Witte, Bd. 7, Schriften zu Literatur, Theater und Kunst. Lyrik, Autobiographie und Drama, hrsg. v. Emily D. Bilski, Heike Breitenbach, Freddie Rokem und Bernd Witte (München: Gütersloher Verlagshaus, 2016), 286.

43 Rosenzweig, Briefe und Tagebücher, Bd. 2, 1120.

44 So z. B. Michael Brocke, „Händel und Bach“, Kalonymos. Beiträge zur deutsch-jüdischen Geschichte aus dem Salomon Ludwig Steinheim-Institut an der Universität Duisburg-Essen 22 (2019), Nr. 1, 16, der den Titel des vorliegenden, auf dem Rosenzweig-Kongress 2019 in Jerusalem vorgetragenen Beitrags vorab aufgreift und aus jüdischer Perspektive kritisch diskutiert. 
werden - und dies trotz des Motuproprios von Pius X. zur heiligen Musik (Inter pastoralis officii sollicitudines, 22. November 1903), trotz der Enzyklika Musicae sacrae disciplina von Pius XII. (25. Dezember 1955), trotz der Konstitution über die heilige Liturgie im Rahmen des II. Vatikanischen Konzils (Sacrosanctum Concilium, 4. Dezember 1963, bes. Kap. VI) und trotz der anschließenden Instruktion Musicam sacram (5. März 1967) des Consilium ad exsequendam Constitutionem de sacra liturgia und der Heiligen Kongregation der Riten. Rosenzweig legt den Akzent jedoch weniger auf die musikalische Form als auf die Funktion der heiligen Musik: Diese ermöglicht zum einen eine Integration kultureller Art, zum anderen ist sie dazu berufen, die Musik zu beleben, da sie nicht zu einem individuellen Genuss, sondern zu einer gemeinsamen Aufführung einlädt. In diesem Sinn ist die liturgische Musik - um bei der katholischen Tradition zu bleiben - nach wie vor ein Mittel des gemeinsamen Gebets, damit die Stimmen der Gläubigen, paradoxerweise auch die schweigenden, erklingen, die in und aus allen Teilen der Welt an der Liturgie teilnehmen. Man denke beispielsweise im 20. Jahrhundert an die Kompositionen des Jesuiten Joseph Gelineau, an die von Jacques Berthier komponierten Gesänge der ökumenischen Gemeinde von Taizé, an die Beatmesse, den Christian rock, die Misa Criolla von Ariel Ramírez und den Sacropop überhaupt.

Doch möchte ich abschließend nicht den musikalisch-liturgischen Aspekt in den Vordergrund stellen. Diesbezüglich sei lediglich der Hinweis gestattet, dass es wirklich lohnen würde, das große Werk Bachs in den Kultstätten erneut aufzuwerten, da es dank seiner Erhabenheit wahrscheinlich imstande ist, die konfessionellen Grenzen zu überwinden. Meine Frage zum Schluss lautet dagegen, ob und wie es heute möglich ist, die Musik der Erlösung mitzusingen oder auch nur mitzuhören im Erleben einer herausragenden Beziehung, die in eine kommende Zeit einführt und ein überweltliches Szenario erschließt, indem sie die Ewigkeit vorwegnimmt.

Auf dem Rosenzweig-Kongress 2017 in Rom wurde von mir die Ansicht vertreten, dass ein bewusstes Leben in der fließenden Postmoderne, in der das Subjekt, rastlos umherirrend und der Entscheidungsmacht über die Ereignisse beraubt, eine Identitätskrise zu durchleben scheint, bedeutet, die Sehnsucht nach etwas zu pflegen, dessen Gestalt man nicht kennt, das aber nichtsdestoweniger in der Form der eigenen Abwesenheit da ist. ${ }^{45}$ Wir sind aufgerufen, auf neue Weise Wanderer zu sein, peregrini im buchstäblichen Sinn (per agros ire) und in gewisser Weise auch im religiösen Sinn (pelegrini, Pilger). Je extremer die Zersplitterung und die Unterschiedlichkeit sind, desto mehr werden die irrenden Subjekte fähig sein, die Sehnsucht nach der Einheit der Form zu verspüren und sie als Aufgabe und

45 Siehe Luca Bertolino, „Rosenzweig in prospettiva. Il nuovo pensiero tra vecchia filosofia e filosofia della differenza“, Archivio di Filosofia 86 (2018), Nr. 1, 221-231: 230 f. 
Zweck ihres Irrens anzunehmen. Umso mehr werden sie außerdem die Erzählungen und Gesänge ähnlicher Erfahrungen wiederzuerkennen verstehen; sie werden das sehnsuchtsvolle Wort vernehmen, das jeweils fragt und stets der Antwort harrt.

Sie werden sich also wie Nomaden an einer erquickenden Oase oder wie Vagabunden am abendlichen Feuer Tausende kleiner und weniger kleiner Geschichten erzählen können, die als Gewebe des Alltagslebens fungieren. Oder sie werden, wie postmoderne Seefahrer, in den Schenken singen, in die sie ihre ungewisse Route zu einem Ziel, das sie nicht kennen, oder einer Insel, von der sie höchstens den Namen kennen, bei ihren verschiedenen Aufenthalten an Land führt. So lese ich, mit einer zum Teil gewagten Interpretation, die Verse, mit denen Konstantinos Kavafis in einem Gedicht von 1911 die Seefahrt nach „Ithaka“ beschreibt, jeder zu seinem eigenen Ithaka:

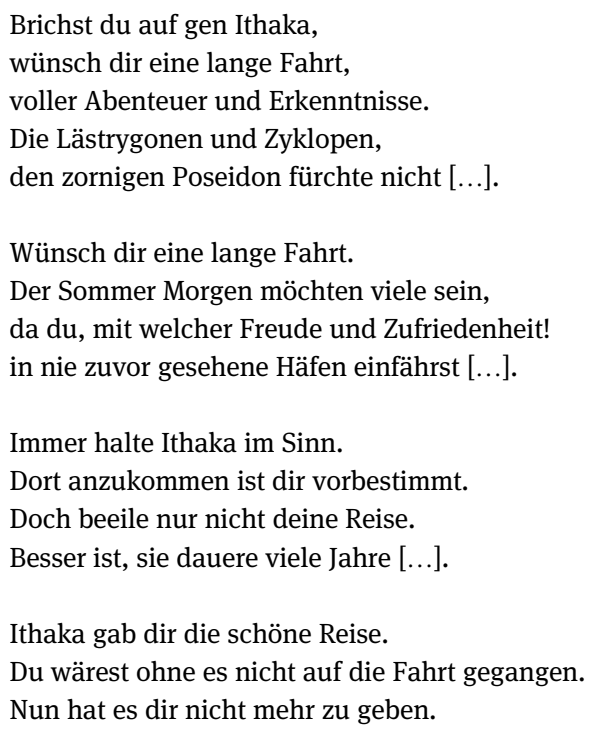

Auch wenn es sich dir ärmlich zeigt, Ithaka betrog dich nicht.

So weise, wie du wurdest, und in solchem Maß erfahren, wirst du ohnedies verstanden haben, was die Ithakas bedeuten. ${ }^{46}$

Kavafis denkt offensichtlich an die Fahrt zu einem bekannten Ziel, aber er führt die Reise dorthin auch als sehnsuchtsvolles, erlebnisreiches Fahren vor, das keine Vergangenheitsnostalgie kennt.

46 Konstantinos Kavafis, „Ithaka“, in: ders., Brichst du auf gen Ithaka ... Sämtliche Gedichte, übersetzt v. Wolfgang Josing unter Mitarbeit v. Doris Gundert (Köln: Romiosini, 1983), 17 f. 
Nicht allein werden die Erzählungen und der Gesang der anderen dergestalt leicht als die eigenen Erzählungen und der eigene Gesang erkennbar, sondern die Sehnsucht dieser weltlichen ekklēsia wird auch auf die Musik des choralen Gebets abfärben und zur Wiederentdeckung der Dimension alter Pilgerfahrten führen. Die Sehnsucht der irrenden Subjekte kann sich nämlich in den Chor derer verwandeln, die in der gemeinsamen Sehnsucht nach der Form, die als abwesende anwesend ist, und sogar in der Hoffnung auf eine Anwesenheit, die die Abwesenheit füllt, leben. Wie Rosenzweig lehrt, wird ein

sich steigernder Gesang [auftreten]. Und Urgesang, der stets Gesang von mehreren ist; der Einzelne singt nicht [...]. [...] [U]rsprünglich ist der Gesang vielstimmig gleichen Tons und Atems, und über allem Inhalt des Gesanges steht die Form dieser Gemeinsamkeit. Ja der Inhalt ist selbst weiter gar nichts als die Begründung für diese seine Form. Man singt nicht gemeinsam um eines bestimmten Inhalts willen, sondern man sucht sich einen gemeinsamen Inhalt, damit man gemeinsam singen kann. ${ }^{47}$

Die Namen finden zusammen in der Hinwendung zur transzendentalen und transzendenten Form, die sie ermöglicht und zugleich übersteigt. Die einzelnen Stimmen werden durch das Duale des gegenseitigen Sich-Anspornens zur Ganzheit des Wir übergehen und sich dem Abwesenden nicht nur nähern, sondern in gewisser Weise auch der Grenzmöglichkeit öffnen, ihm zu begegnen. Es ist zum Beispiel die Musik der Vorläufigkeit, aber auch der Gastfreundschaft, die man empfangen und schenken kann, die Musik der Mühe, aber auch der Freude am Teilen, die Musik der Armut, aber auch des Reichtums, den der andere darstellt. Es ist eine Musik, die gesungen werden will, die mitreißt und überflutet. Erlösung geschieht.

Aus dem Italienischen von Leonie Schröder

47 Rosenzweig, Der Stern der Erlösung, 258. 